\title{
Unit Testing of ETSI EVS Codec
}

\author{
Arvind Vishnubhatla \\ Electronics and Communication Department \\ Gokaraju Rangaraju institute of Engineering and Technology \\ Hyderabad, India
}

\begin{abstract}
As a part of software development process, the small testable parts are individually tested for correctness.

A superwideband audio coding standard called Enhanced Voice services provides $20 \mathrm{kHz}$ audio bandwidth. The standard intends to provide robustness to delay jitter and packet losses as the coding is channel aware.

Here the problem at hand is the EVS Codec code provided by ETSI. The encoder and decoder c code is made in linux into executable files and sample linux scripts are used determine the correctness of coding and decoding.
\end{abstract}

The successful running of test ensures quality of product.

\section{General Terms}

UMTS: Universal Mobile Telecommunications System

EVS: Codec for Enhanced Voice Services

\section{Keywords}

EVS, superwideband, bandwidth, Codec, unit testing.

\section{INTRODUCTION}

As a part of software development process, the small testable parts are individually tested for correctness.

A superwideband audio coding standard called Enhanced Voice services provides $20 \mathrm{kHz}$ audio bandwidth. The standard intends to provide robustness to delay jitter and packet losses as the coding is channel aware.

To considerably improve network capacity and voice quality [1], 3GPP has standardized voice services. The quality of speech rendered matches that of stored music.

The codec has a lot of new features which improve operation in harsh environments. The Codec makes use of ACELP and MDCT to switch between speech and audio compression modes. A collaborative effort amongst handset and infrastructure manufacturers resulted in the evolution of the standard [2].

The codec gives higher quality, better frame/packet error resilience and provides better compression efficiency. [4]

The codec is cost effective and provides better connectivity. In the previous years, only a small part of the dynamic range of human voice was transmitted. With the emergence of this codec it looks as if we are talking face to face with the other person.

Here the problem at hand is the EVS Codec code provided by ETSI. The encoder and decoder c code is made in linux into executable files and sample linux scripts are used determine the correctness of coding and decoding.

Although the technical rigor of understanding and coding the standard is immense, the level of testing [5] where individual components are validated is by no means trivial.
Each of the individual program modules are tested by creating representative meaningful test stimuli and the outcome is verified against an already available benchmark. [6]

The goal of unit testing is to isolate each part of the program and ensure that individual parts are working correctly. [7]

Sometimes developers try to cut costs by performing minimal testing which introduces defects which percolate through the final product. [8]

This increases the cost of system testing, alpha and beta testing. [9]

\section{THE MAKE PROCESS}

The code is loaded in a Linux machine and a standard makefile is used to compile the code. The targets EVS_cod and EVS_dec are made

Compiling lib_enc/ACcontextMapping_enc.c

Compiling lib_enc/acelp_core_enc.c

Compiling lib_enc/acelp_core_switch_enc.c

Compiling lib_enc/acelp_enc_util.c

Compiling lib_enc/amr_wb_enc.c

Compiling lib_enc/analy_lp.c

Compiling lib_enc/analy_sp.c

Compiling lib_enc/ari_enc.c

Compiling lib_enc/ari_hm_enc.c

Compiling lib_enc/arith_coder_enc.c

Compiling lib_enc/avq_cod.c

Compiling lib_enc/bass_psfilter_enc.c

Compiling lib_enc/bw_detect.c

Compiling lib_enc/cng_enc.c

Compiling lib_enc/cod2t32.c

Compiling lib_enc/cod4t64.c

Compiling lib_enc/cod_ace.c

Compiling lib_enc/cod_tcx.c

Compiling lib_enc/cod_uv.c

Compiling lib_enc/comvad_decision.c

Compiling lib_enc/core_enc_2div.c

Compiling lib_enc/core_enc_init.c

Compiling lib_enc/core_enc_ol.c

Compiling lib_enc/core_enc_reconf.c

Compiling lib_enc/core_enc_switch.c

Compiling lib_enc/core_enc_updt.c 
Compiling lib_enc/core_switching_enc.c

Compiling lib_enc/corr_xh.c

Compiling lib_enc/cor_shif.c

Compiling lib_enc/decision_matrix_enc.c

Compiling lib_enc/detect_transient.c

Compiling lib_enc/diffcod.c

Compiling lib_enc/dtx.c

Compiling lib_enc/enc_acelp.c

Compiling lib_enc/enc_acelp_tcx_main.c

Compiling lib_enc/enc_acelpx.c

Compiling lib_enc/enc_amr_wb.c

Compiling lib_enc/enc_gain.c

Compiling lib_enc/enc_gen_voic.c

Compiling lib_enc/enc_gen_voic_rf.c

Compiling lib_enc/enc_higher_acelp.c

Compiling lib_enc/enc_nelp.c

Compiling lib_enc/encoder.c

Compiling lib_enc/enc_pit_exc.c

Compiling lib_enc/enc_ppp.c

Compiling lib_enc/enc_prm.c

Compiling lib_enc/enc_tran.c

Compiling lib_enc/enc_uv.c

Compiling lib_enc/energy.c

Compiling lib_enc/eval_pit_contr.c

Compiling lib_enc/evs_enc.c

Compiling lib_enc/ext_sig_ana.c

Compiling lib_enc/fd_cng_enc.c

Compiling lib_enc/FEC_enc.c

Compiling lib_enc/find_tar.c

Compiling lib_enc/find_tilt.c

Compiling lib_enc/find_uv.c

Compiling lib_enc/find_wsp.c

Compiling lib_enc/frame_spec_dif_cor_rate.c

Compiling lib_enc/gain_enc.c

Compiling lib_enc/gaus_enc.c

Compiling lib_enc/gp_clip.c

Compiling lib_enc/gs_enc.c

Compiling lib_enc/guided_plc_enc.c

Compiling lib_enc/hf_cod_amrwb.c

Compiling lib_enc/hq_classifier_enc.c

Compiling lib_enc/hq_core_enc.c

Compiling lib_enc/hq_env_enc.c

Compiling lib_enc/hq_hr_enc.c
Compiling lib_enc/hq_lr_enc.c

Compiling lib_enc/hvq_enc.c

Compiling lib_enc/igf_enc.c

Compiling lib_enc/igf_scf_enc.c

Compiling lib_enc/init_enc.c

Compiling lib_enc/inov_enc.c

Compiling lib_enc/io_enc.c

Compiling lib_enc/isf_enc_amr_wb.c

Compiling lib_enc/lead_indexing.c

Compiling lib_enc/long_enr.c

Compiling lib_enc/lp_exc_e.c

Compiling lib_enc/lsf_enc.c

Compiling lib_enc/lsf_msvq_ma_enc.c

Compiling lib_enc/ltd_stable.c

Compiling lib_enc/mdct_classifier.c

Compiling lib_enc/mdct_selector.c

Compiling lib_enc/mslvq_enc.c

Compiling lib_enc/multi_harm.c

Compiling lib_enc/nelp_enc.c

Compiling lib_enc/noise_adjust.c

Compiling lib_enc/nois_est.c

Compiling lib_enc/normalizecoefs.c

Compiling lib_enc/peak_vq_enc.c

Compiling lib_enc/pitch_ol2.c

Compiling lib_enc/pitch_ol.c

Compiling lib_enc/pit_enc.c

Compiling lib_enc/plc_enc_ext.c

Compiling lib_enc/ppp_enc.c

Compiling lib_enc/pre_proc.c

Compiling lib_enc/pvq_core_enc.c

Compiling lib_enc/pvq_encode.c

Compiling lib_enc/q_gain2p.c

Compiling lib_enc/qlpc_avq.c

Compiling lib_enc/qlpc_stoch.c

Compiling lib_enc/range_enc.c

Compiling lib_enc/re8_cod.c

Compiling lib_enc/reordernorm.c

Compiling lib_enc/rom_enc.c

Compiling lib_enc/rst_enc.c

Compiling lib_enc/set_impulse.c

Compiling lib_enc/setmodeindex.c

Compiling lib_enc/sig_clas.c

Compiling lib_enc/SNR_calc.c 
Compiling lib_enc/spec_center.c

Compiling lib_enc/spec_flatness.c

Compiling lib_enc/speech_music_classif.c

Compiling lib_enc/stat_noise_uv_enc.c

Compiling lib_enc/subband_fft.c

Compiling lib_enc/swb_bwe_enc.c

Compiling lib_enc/swb_bwe_enc_hr.c

Compiling lib_enc/swb_bwe_enc_lr.c

Compiling lib_enc/swb_pre_proc.c

Compiling lib_enc/swb_tbe_enc.c

Compiling lib_enc/tcq_core_enc.c

Compiling lib_enc/tcx_ltp_enc.c

Compiling lib_enc/tcx_utils_enc.c

Compiling lib_enc/tfa_enc.c

Compiling lib_enc/tns_base_enc.c

Compiling lib_enc/transient_detection.c

Compiling lib_enc/transition_enc.c

Compiling lib_enc/update_decision.c

Compiling lib_enc/updt_enc.c

Compiling lib_enc/updt_tar.c

Compiling lib_enc/vad.c

Compiling lib_enc/vad_param_updt.c

Compiling lib_enc/vad_proc.c

Compiling lib_enc/vbr_average_rate.c

Compiling lib_enc/vlpc_1st_cod.c

Compiling lib_enc/vlpc_2st_cod.c

Compiling lib_enc/voiced_enc.c

Compiling lib_enc/waveadjust_fec_cod.c

Compiling lib_com/ACcontextMapping.c

Compiling lib_com/ari.c

Compiling lib_com/ari_hm.c

Compiling lib_com/arith_coder.c

Compiling lib_com/basop32.c

Compiling lib_com/basop_com_lpc.c

Compiling lib_com/basop_lsf_tools.c

Compiling lib_com/basop_mpy.c

Compiling lib_com/basop_tcx_utils.c

Compiling lib_com/basop_util.c

Compiling lib_com/bitalloc.c

Compiling lib_com/bitallocsum.c

Compiling lib_com/bits_alloc.c

Compiling lib_com/bitstream.c

Compiling lib_com/calc_st_com.c
Compiling lib_com/cb_shape.c

Compiling lib_com/cldfb.c

Compiling lib_com/cng_exc.c

Compiling lib_com/codec_tcx_common.c

Compiling lib_com/core_com_config.c

Compiling lib_com/deemph.c

Compiling lib_com/delay_comp.c

Compiling lib_com/disclaimer.c

Compiling lib_com/dlpc_bfi.c

Compiling lib_com/edct.c

Compiling lib_com/enh1632.c

Compiling lib_com/enh40.c

Compiling lib_com/enhancer.c

Compiling lib_com/enr_1_az.c

Compiling lib_com/env_adj.c

Compiling lib_com/env_stab.c

Compiling lib_com/env_stab_trans.c

Compiling lib_com/est_tilt.c

Compiling lib_com/fd_cng_com.c

Compiling lib_com/fft.c

Compiling lib_com/fft_cldfb.c

Compiling lib_com/fft_rel.c

Compiling lib_com/fill_spectrum.c

Compiling lib_com/findpulse.c

Compiling lib_com/fine_gain_bits.c

Compiling lib_com/frame_ener.c

Compiling lib_com/g192.c

Compiling lib_com/get_gain.c

Compiling lib_com/gs_bitallocation.c

Compiling lib_com/gs_gains.c

Compiling lib_com/gs_inact_switching.c

Compiling lib_com/gs_noisefill.c

Compiling lib_com/gs_preech.c

Compiling lib_com/guided_plc_util.c

Compiling lib_com/hp50.c

Compiling lib_com/hq2_bit_alloc.c

Compiling lib_com/hq2_core_com.c

Compiling lib_com/hq2_noise_inject.c

Compiling lib_com/hq_bit_allocation.c

Compiling lib_com/hq_conf.c

Compiling lib_com/hq_tools.c

Compiling lib_com/hvq_pvq_bitalloc.c

Compiling lib_com/ifft_rel.c 
Compiling lib_com/igf_base.c

Compiling lib_com/index_pvq_opt.c

Compiling lib_com/interleave_spectrum.c

Compiling lib_com/interpol.c

Compiling lib_com/int_lsp.c

Compiling lib_com/isf_dec_amr_wb.c

Compiling lib_com/lag_wind.c

Compiling lib_com/lerp.c

Compiling lib_com/limit_t0.c

Compiling lib_com/logqnorm.c

Compiling lib_com/longarith.c

Compiling lib_com/low_rate_band_att.c

Compiling lib_com/lpc_tools.c

Compiling lib_com/lsf_dec_bfi.c

Compiling lib_com/lsf_msvq_ma.c

Compiling lib_com/lsf_tools.c

Compiling lib_com/lsp_conv_poly.c

Compiling lib_com/modif_fs.c

Compiling lib_com/mslvq_com.c

Compiling lib_com/nelp.c

Compiling lib_com/parameter_bitmaping.c

Compiling lib_com/phase_dispersion.c

Compiling lib_com/ppp.c

Compiling lib_com/pred_lt4.c

Compiling lib_com/preemph.c

Compiling lib_com/pvq_com.c

Compiling lib_com/range_com.c

Compiling lib_com/re8_ppv.c

Compiling lib_com/re8_util.c

Compiling lib_com/realft.c

Compiling lib_com/recovernorm.c

Compiling lib_com/reordvct.c

Compiling lib_com/residu.c

Compiling lib_com/rom_com.c

Compiling lib_com/stab_est.c

Compiling lib_com/stat_noise_uv_mod.c

Compiling lib_com/swb_bwe_com.c

Compiling lib_com/swb_bwe_com_hr.c

Compiling lib_com/swb_bwe_com_lr.c

Compiling lib_com/swb_tbe_com.c

Compiling lib_com/syn_12k8.c

Compiling lib_com/syn_filt.c

Compiling lib_com/tcq_position_arith.c
Compiling lib_com/tcx_ltp.c

Compiling lib_com/tcx_mdct.c

Compiling lib_com/tcx_mdet_window.c

Compiling lib_com/tcx_utils.c

Compiling lib_com/tec_com.c

Compiling lib_com/tns_base.c

Compiling lib_com/tools.c

Compiling lib_com/trans_direct.c

Compiling lib_com/trans_inv.c

Compiling lib_com/vlpc_2st_com.c

Compiling lib_com/weight_a.c

Compiling lib_com/weight.c

Compiling lib_com/wi.c

Compiling lib_com/window.c

Compiling lib_com/window_ola.c

Compiling lib_com/wtda.c

Linking EVS_cod

Compiling lib_dec/ACcontextMapping_dec.c

Compiling lib_dec/acelp_core_dec.c

Compiling lib_dec/acelp_core_switch_dec.c

Compiling lib_dec/amr_wb_dec.c

Compiling lib_dec/ari_dec.c

Compiling lib_dec/ari_hm_dec.c

Compiling lib_dec/arith_coder_dec.c

Compiling lib_dec/avq_dec.c

Compiling lib_dec/bass_psfilter.c

Compiling lib_dec/cng_dec.c

Compiling lib_dec/core_dec_init.c

Compiling lib_dec/core_dec_reconf.c

Compiling lib_dec/core_dec_switch.c

Compiling lib_dec/core_switching_dec.c

Compiling lib_dec/dec2t32.c

Compiling lib_dec/dec4t64.c

Compiling lib_dec/dec_ace.c

Compiling lib_dec/dec_acelp.c

Compiling lib_dec/dec_acelp_tcx_main.c

Compiling lib_dec/dec_amr_wb.c

Compiling lib_dec/dec_gen_voic.c

Compiling lib_dec/dec_higher_acelp.c

Compiling lib_dec/decision_matrix_dec.c

Compiling lib_dec/dec_LPD.c

Compiling lib_dec/dec_nelp.c

Compiling lib_dec/decoder.c 
Compiling lib_dec/dec_pit_exc.c

Compiling lib_dec/dec_post.c

Compiling lib_dec/dec_ppp.c

Compiling lib_dec/dec_prm.c

Compiling lib_dec/dec_tcx.c

Compiling lib_dec/dec_tran.c

Compiling lib_dec/dec_uv.c

Compiling lib_dec/d_gain2p.c

Compiling lib_dec/dlpc_avq.c

Compiling lib_dec/dlpc_stoch.c

Compiling lib_dec/er_dec_acelp.c

Compiling lib_dec/er_dec_tcx.c

Compiling lib_dec/er_scale_syn.c

Compiling lib_dec/er_sync_exc.c

Compiling lib_dec/er_util.c

Compiling lib_dec/evs_dec.c

Compiling lib_dec/EvsRXlib.c

Compiling lib_dec/fd_cng_dec.c

Compiling lib_dec/FEC_adapt_codebook.c

Compiling lib_dec/FEC.c

Compiling lib_dec/FEC_clas_estim.c

Compiling lib_dec/FEC_HQ_core.c

Compiling lib_dec/FEC_HQ_phase_ecu.c

Compiling lib_dec/FEC_lsf_estim.c

Compiling lib_dec/FEC_pitch_estim.c

Compiling lib_dec/FEC_scale_syn.c

Compiling lib_dec/gain_dec.c

Compiling lib_dec/gaus_dec.c

Compiling lib_dec/gs_dec_amr_wb.c

Compiling lib_dec/gs_dec.c

Compiling lib_dec/hdecnrm.c

Compiling lib_dec/hf_synth.c

Compiling lib_dec/hq_classifier_dec.c

Compiling lib_dec/hq_conf_fec.c

Compiling lib_dec/hq_core_dec.c

Compiling lib_dec/hq_env_dec.c

Compiling lib_dec/hq_hr_dec.c

Compiling lib_dec/hq_lr_dec.c

Compiling lib_dec/igf_dec.c

Compiling lib_dec/igf_scf_dec.c

Compiling lib_dec/init_dec.c

Compiling lib_dec/inov_dec.c

Compiling lib_dec/io_dec.c
Compiling lib_dec/jbm_jb4_circularbuffer.c

Compiling lib_dec/jbm_jb4_inputbuffer.c

Compiling lib_dec/jbm_jb4_jmf.c

Compiling lib_dec/jbm_jb4sb.c

Compiling lib_dec/jbm_pcmdsp_apa.c

Compiling lib_dec/jbm_pcmdsp_fifo.c

Compiling lib_dec/jbm_pcmdsp_similarityestimation.c

Compiling lib_dec/jbm_pcmdsp_window.c

Compiling lib_dec/LD_music_post_filter.c

Compiling lib_dec/lead_deindexing.c

Compiling lib_dec/lp_exc_d.c

Compiling lib_dec/lsf_dec.c

Compiling lib_dec/lsf_msvq_ma_dec.c

Compiling lib_dec/nelp_dec.c

Compiling lib_dec/peak_vq_dec.c

Compiling lib_dec/pitch_extr.c

Compiling lib_dec/pit_dec.c

Compiling lib_dec/post_dec.c

Compiling lib_dec/ppp_dec.c

Compiling lib_dec/pvq_core_dec.c

Compiling lib_dec/pvq_decode.c

Compiling lib_dec/range_dec.c

Compiling lib_dec/re8_dec.c

Compiling lib_dec/rom_dec.c

Compiling lib_dec/rst_dec.c

Compiling lib_dec/stat_noise_uv_dec.c

Compiling lib_dec/swb_bwe_dec.c

Compiling lib_dec/swb_bwe_dec_hr.c

Compiling lib_dec/swb_bwe_dec_lr.c

Compiling lib_dec/swb_tbe_dec.c

Compiling lib_dec/syn_outp.c

Compiling lib_dec/tcq_core_dec.c

Compiling lib_dec/tcx_utils_dec.c

Compiling lib_dec/tns_base_dec.c

Compiling lib_dec/TonalComponentDetection.c

Compiling lib_dec/tonalMDCTconcealment.c

Compiling lib_dec/transition_dec.c

Compiling lib_dec/updt_dec.c

Compiling lib_dec/vlpc_1st_dec.c

Compiling lib_dec/vlpc_2st_dec.c

Compiling lib_dec/voiced_dec.c

Compiling lib_dec/voip_client.c

Compiling lib_dec/waveadjust_fec_dec.c 
Linking EVS_dec

\section{THE ENCODE PROCESS}

\subsection{The organization of source and input test files.}

A set of input audio files are properly prepared and stored in the test directory

\section{[ rootevlsibtechclient26 +loatc]\# Is \\ bin encodscripti lib_enc switchPaths \\ build Evs cod Makefile test \\ copyscript Eus cod,exe output testw \\ decodscript Eus dec Readme EVS dec log.tXt Workspace_msve \\ decodscriptil Eus dec, exe Readme Evs enci log.txt \\ enclog lib_com Readme Evs enc log.tot \\ encodscript lib dec readme.txt \\ [rootQvilsibtechclient26 floatc]\#]}

Fig 1: A snapshot of the directory structure of the codec files.

The directory lib_enc contains c source files for the encoder which forms the acelp and mdct part.

The source files for the decoder are stored in the directory lib_dec.

The common files are stored in lib_com directory.

A Makefile is crafted to compile the encoder and decoder.

To test the codec a series of input speech files are created and stored as subdirectories under the directory testv.

\section{[rootavisibtechlclient26 testv]|\# Is}

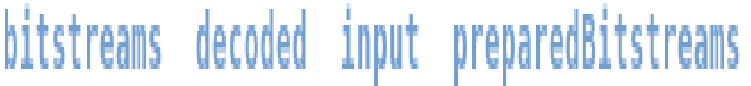



Fig 2: A subset of directories which contain the input speech files.

[rootevlsibtechclient26 testv]\# \s

bitstreams decoded input preparedBitstreams

[rootevlsibtechclient26 testv]\# col bitstreams/

[rootevlsibtechclient26 bitstreams]\# Is

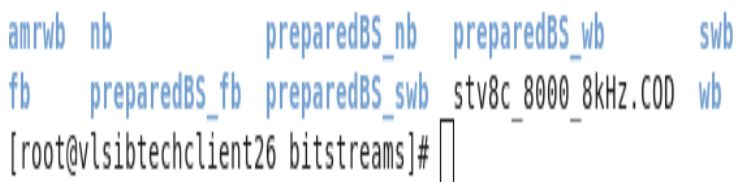

Fig 2: Different directories of input speech files amrwb, narrowband, wideband superwideband are generated and stored in different directories.

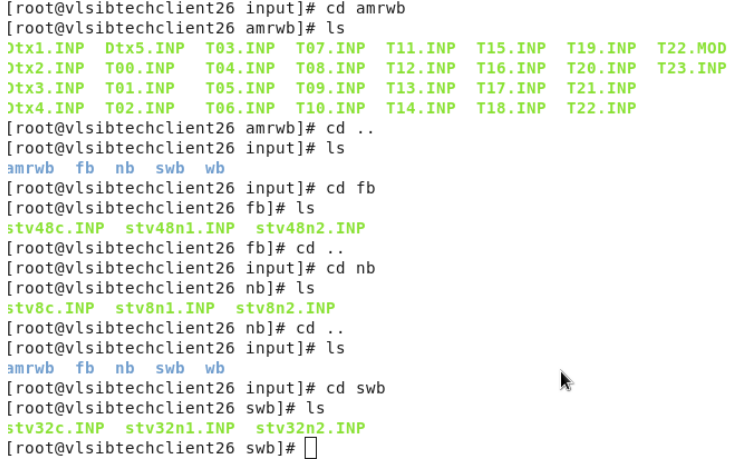

Fig 3: A listing different INP files which are speech files.

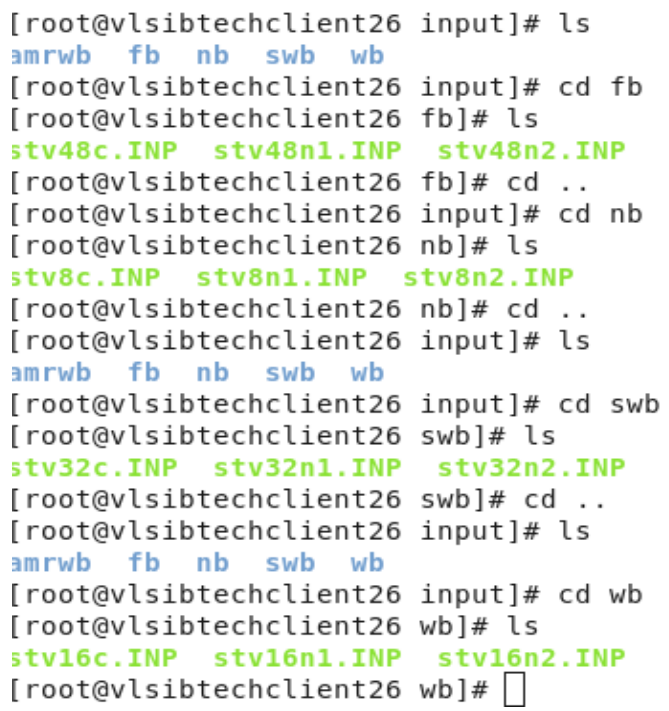

Fig 4: A listing different INP files for different speech formats.

\subsection{The encoding script}

Now the encoding process is initiated by passing the input files one after the other on the command line to the input codec and the result is obtained as *.COD file. The entire process is encoded as a script. A part of the script is shown here for reference.

ENC_BIN=./EVS_cod \# EVS encoder binary under test

DIFF_BIN=/usr/bin/diff \# default test tool

LOG_FILE=Readme_EVS_enc_log.txt \# test result log file

if [ -f \$LOG_FILE ] ; then

rm \$LOG_FILEfi

\$ENC_BIN ./switchPaths/sw_72_244.bin 8

./testv/input/nb/stv8c.INP

./output/bitstreams/stv8c_sw_72_244_8kHz.COD

\$DIFF_BIN

./testv/bitstreams/nb/stv8c_sw_72_244_8kHz.COD

./output/bitstreams/stv8c_sw_72_244_8kHz.COD >>

\$LOG_FILE 2>\&1

\$ENC_BIN $72008 . /$ testv/input/nb/stv8c.INP ./output/bitstreams/stv8c_7200_8kHz.COD

\$DIFF_BIN ./testv/bitstreams/nb/stv8c_7200_8kHz.COD ./output/bitstreams/stv8c_7200_8kHz.COD $>$ \$ \$LOG_FILE $2>\& 1$ 
\$ENC_BIN 80008 ./testv/input/nb/stv8c.INP ./output/bitstreams/stv8c_8000_8kHz.COD

\$DIFF_BIN ./testv/bitstreams/nb/stv8c_8000_8kHz.COD ./output/bitstreams/stv8c_8000_8kHz.COD > \$LOG_FILE $2>\& 1$

\$ENC_BIN $96008 . /$ testv/input/nb/stv8c.INP ./output/bitstreams/stv8c_9600_8kHz.COD

\$DIFF_BIN ./testv/bitstreams/nb/stv8c_9600_8kHz.COD ./output/bitstreams/stv8c_9600_8kHz.COD $>$ > \$LOG_FILE $2>\& 1$

\$ENC_BIN -dtx $59008 . /$ testv/input/nb/stv8c.INP ./output/bitstreams/stv8c_dtx_5900_8kHz.COD

\$DIFF_BIN ./testv/bitstreams/nb/stv8c_dtx_5900_8kHz.COD ./output/bitstreams/stv8c_dtx_5900_8kHz.COD >> \$LOG_FILE 2>\&1

\$ENC_BIN $132008 . /$ testv/input/nb/stv8c.INP ./output/bitstreams/stv8c_13200_8kHz.COD

\$DIFF_BIN ./testv/bitstreams/nb/stv8c_13200_8kHz.COD ./output/bitstreams/stv8c_13200_8kHz.COD $>$ \$LOG_FILE $2>\& 1$

\section{THE DECODE PROCESS}

Now the decoding process is initiated by passing the encoded *.COD through the EVS decoder to produce *.OUT files. These OUT files are compared to an existing benchmark file using the diff command. A portion of the decoder script is attached for reference.

\subsection{The decoding script}

DEC_BIN=./EVS_dec \# EVS decoder binary under test

DIFF_BIN=diff \# default test tool

LOG_FILE=Readme_EVS_dec_log.txt \# test result log file

if [ -f \$LOG_FILE]; then

rm \$LOG_FILEfi

\section{\$DEC_BIN 8}

.testv/bitstreams/nb/stv8c_13200_8kHz.f06.COD

./output/decoded/stv8c_13200_8kHz.f06.OUT

\$DIFF_BIN ./testv/decoded/nb/stv8c_13200_8kHz.f06.OUT ./output/decoded/stv8c_13200_8kHz.f06.OUT >> \$LOG_FILE 2>\&1

\$DEC_BIN 8

./testv/bitstreams/nb/stv8c_13200_8kHz.COD

./output/decoded/stv8c_13200_8kHz.OUT

\$DIFF_BIN ./testv/decoded/nb/stv8c_13200_8kHz.OUT ./output/decoded/stv8c_13200_8kHz.OUT >> \$LOG_FILE $2>\& 1$

\$DEC_BIN 8

./testv/bitstreams/nb/stv8c_13200_8kHz.b10.COD

./output/decoded/stv8c_13200_8kHz.b10.OUT

\$DIFF_BIN ./testv/decoded/nb/stv8c_13200_8kHz.b10.OUT ./output/decoded/stv8c_13200_8kHz.b10.OUT >> \$LOG_FILE 2>\&1

\$DEC BIN 8

./testv/bitstreams/nb/stv8c 8000 8kHz.b10.COD

./output/decoded/stv8c_8000_8kHz.b10.OUT
\$DIFF_BIN ./testv/decoded/nb/stv8c_8000_8kHz.b10.OUT ./output/decoded/stv8c_8000_8kHz.b10.OUT >> \$LOG_FILE $2>\& 1$

\section{THE UNIT TESTING PROCESS}

A portion of the encoding $\log$ as a part of encoding is as shown below:

EVS Codec (Floating Point) 3GPP TS26.443 Apr 03, 2018. Version 12.10.0 / 13.6.0 / 14.2.0

Bandwidth switching file:

./switchPaths/bw_switch_NB_WB_SWB_FB.txt

Bitrate switching file: ./switchPaths/bw_59_1280.bin

Input audio file: ./testv/input/fb/stv48n2.INP

Output bitstream file:

./output/bitstreams/stv48n2_bw_59_1280_48kHz.COD

Input sampling rate: $48000 \mathrm{~Hz}$

Bitrate: $\quad 7.20 \mathrm{kbps}$

Bandwidth limited to WB. To enable FB coding, please use max_band FB.

Running the encoder ------

Frames processed: $\quad 700$

Encoding finished

At the end of the total encoding and decoding process we get the $\log$

\section{TEST PASSED SUCESSFULLY}

\section{DISCUSSION}

Even when performing unit testing the task should not be considered as trivial. The quality of the output is dependent on how meticulously the unit test benchmarks are created. This process even though simple illustrates that unit testing is by no means a simple task. Lack of proper planning can result in a faulty product being handed over to the end customer

\section{REFERENCES}

[1] Universal Mobile Telecommunications System (UMTS); LTE; Codec for Enhanced Voice Services (EVS); ANSI C code (fixed-point) (3GPP TS 26.442 version 14.2.0 Release 14).

[2] Atti, V.; Sinder, D. J.; Subasingha, S.; Rajendran, V.; Dewasurendra, D.; Chebiyyam, V.; Varga, I.; Krishnan, V.; Schubert, B. (2015-04-01). "Improved error resilience for volte and VoIP with 3GPP EVS channel aware coding". 2015 IEEE International Conference on Acoustics, Speech and Signal Processing (ICASSP): 5713-5717. doi:10.1109/ICASSP.2015.7179066

[3] Lecomte, J.; Vaillancourt, T.; Bruhn, S.; Sung, H.; Peng, K.; Kikuiri, K.; Wang, B.; Subasingha, S.; Faure, J. (2015-04-01). "Packet-loss concealment technology advances in EVS". 2015 IEEE International Conference on Acoustics, Speech and Signal Processing (ICASSP): 5708-5712. doi:10.1109/ICASSP.2015.7179065.

[4] The 3GPP Enhanced Voice Services (EVS) codec. Nokia white paper.

[5] Kolawa, Adam; Huizinga, Dorota (2007). Automated Defect Prevention: Best Practices in Software Management. Wiley-IEEE Computer Society Press. 
p. 75. ISBN 0-470-04212-5. Klaus David ,Alexander Flach, CAR-2-X and Pedestrian IEEE Vehicular Technology Magazine Page(s): 70 - 76.

[6] Xie, Tao. "Towards a Framework for Differential Unit Testing of Object-Oriented Programs" (PDF). Retrieved 2012-07-23.Tavel, P. 2007 Modeling and Simulation Design. AK Peters Ltd.

[7] "Guide to Agile Practices". Archived from the original on 2012-04-29. Retrieved 2014-01-06. Hojin Jung,Seong
Kyung Kwon,Haengju Lee,Sang Hyuk Son, "Traffic Caution System for Pedestrian Safety," 2016 IEEE 22nd International Conference on Embedded and Real-Time Computing Systems and Applications (RTCSA), DOI: 10.1109/RTCSA.2016.60.

[8] "ISTQB Exam Certification". ISTQB Exam Certification. Retrieved 12 March 2015.

[9] Fowler, Martin (2007-01-02). "Mocks aren't Stubs". Retrieved 2008-04-01. 